\title{
Evaluation of OFU Techniques for Optical Generation of mm-W Signals in Future Broadband Cellular Communication Networks
}

\author{
${ }^{1}$ Nael A. Al-Shareefi, ${ }^{2}$ Mohanad S. Alkhazraj, ${ }^{3}$ Sarah Yahya Ali Al-Abbasi and ${ }^{1}$ Sura Adil Abbas \\ ${ }^{1}$ College of Engineering, University of Information Technology and \\ Communications (UOITC), Baghdad, Iraq \\ ${ }^{2}$ Department of Electrical and Electronic Engineering, University of Technology (UOT), \\ Baghdad, Iraq \\ ${ }^{3}$ Dijlah University College, Baghdad, Iraq
}

\begin{abstract}
Cellular networks that use the millimeter-Wave (mm-W) spectrum holds a great promise to revolutionize wireless industry. Nevertheless, the generation and transportation of $\mathrm{mm}-\mathrm{W}$ signals in the electrical-domain are unfeasible owing to the high air link loss and electromagnetic interference. Generating mm-W signals in the optical-domain, i.e., over an optical fiber will allow sending wireless signals for a long distance. This study presents an evaluation of the Optical Frequency Upconversion (OFU) techniques based on advanced Mach-Zehnder Modulator (MZM) for optical generation and transportation of mm-W wireless signals. Four types of OFU techniques will be compared: frequency-doubling, frequency-quadrupling, frequency-sixtupling and frequency-octupling. We begin by reviewing selected OFU techniques that enable to double, quadruple, sixtuple or octuple an Radio Frequency $(\mathrm{RF})$ and highlighting drawbacks and benefits. Then, a comparison in terms of modulation index, bias drifting and modulation efficiency is presented to show the trade-off between OFU techniques complexity and cost. Comparison of OFU techniques and results from simulation analysis reveal that the frequency quadrupling technique is a robust and more desirable choice for optical generation and transportation of $\mathrm{mm}-\mathrm{W}$ wireless signals.
\end{abstract}

Key words: $\mathrm{OFU}$ techniques, optical mm-W signal generation, $5 \mathrm{G}$, frequency quadrupling, MZM, wireless signals

\section{INTRODUCTION}

Industries and researchers have identified $5 \mathrm{G}$ of cellular technology that will use mm-W band as a key enabler to offer unprecedented wireless access services. Bandwidth-hungry applications like high definition telepresence are now available and need huge bandwidth (Gubbia et al., 2013). These applications force the requirement for a link that can carry high data rates. The usage of microwave frequency range leads to an increase in the amount of traffic congestion which increases the time delay ( $\mathrm{Li}$ et al., 2016). Moreover, the microwave signal is not preferred for long-distance transmission because of fading effect and low sampling rate (Khawaja and Cryan, 2008). The $5 \mathrm{G} \mathrm{mm-W}$ wireless channel bandwidths will be 10 times more than today's $4 \mathrm{G}$ LTE $20-\mathrm{MHz}$ cellular channels in addition it is a promising solution to the spectral congestion (Rappaport et al., 2017).

The optical generation techniques of mm-W signals can be classified into Optical Heterodyne Detection (OHD) (Kitayama et al., 1996; Kuri et al., 2003;
Chuyanov et al., 2005; Hyodo et al., 2009) and OFU (Mohamed et al., 2007; Al-Shareefi et al., 2013a, b) techniques. OFU is a technique wherein high-order optical sidebands are produced utilizing a CW laser with an external optical modulator driven by a sinusoidal $\mathrm{RF}$ signal. By multiplying any two high-order optical sidebands in the $\mathrm{PD}$, numerous mm-W signals may be produced. Compared with the OHD technique, OFU can generate a high quality optical mm-W signals without the use of complex optical injection locking and optical phase locked loop mechanisms for phase noise suppression.

In this study, we have demonstrated an evaluation of OFU techniques for the optical generation of $\mathrm{mm}-\mathrm{W}$ wireless signals. We have also investigated the puirty of the generated optical mm-W signals for selected OFU techniques considering the impact of non-ideal phase difference between RF-driven signals and non-ideal MZM Extinction Ratio (ER).

Electrical approaches for $\mathrm{mm}-\mathrm{W}$ wireless signals transportation: The electrical transportation of $\mathrm{mm}-\mathrm{W}$ wireless signals presents a challenge due to high cost and

Corresponding Author: Nael A. Al-Shareefi, College of Engineering,

University of Information Technology and Communications (UOITC), Baghdad, Iraq 


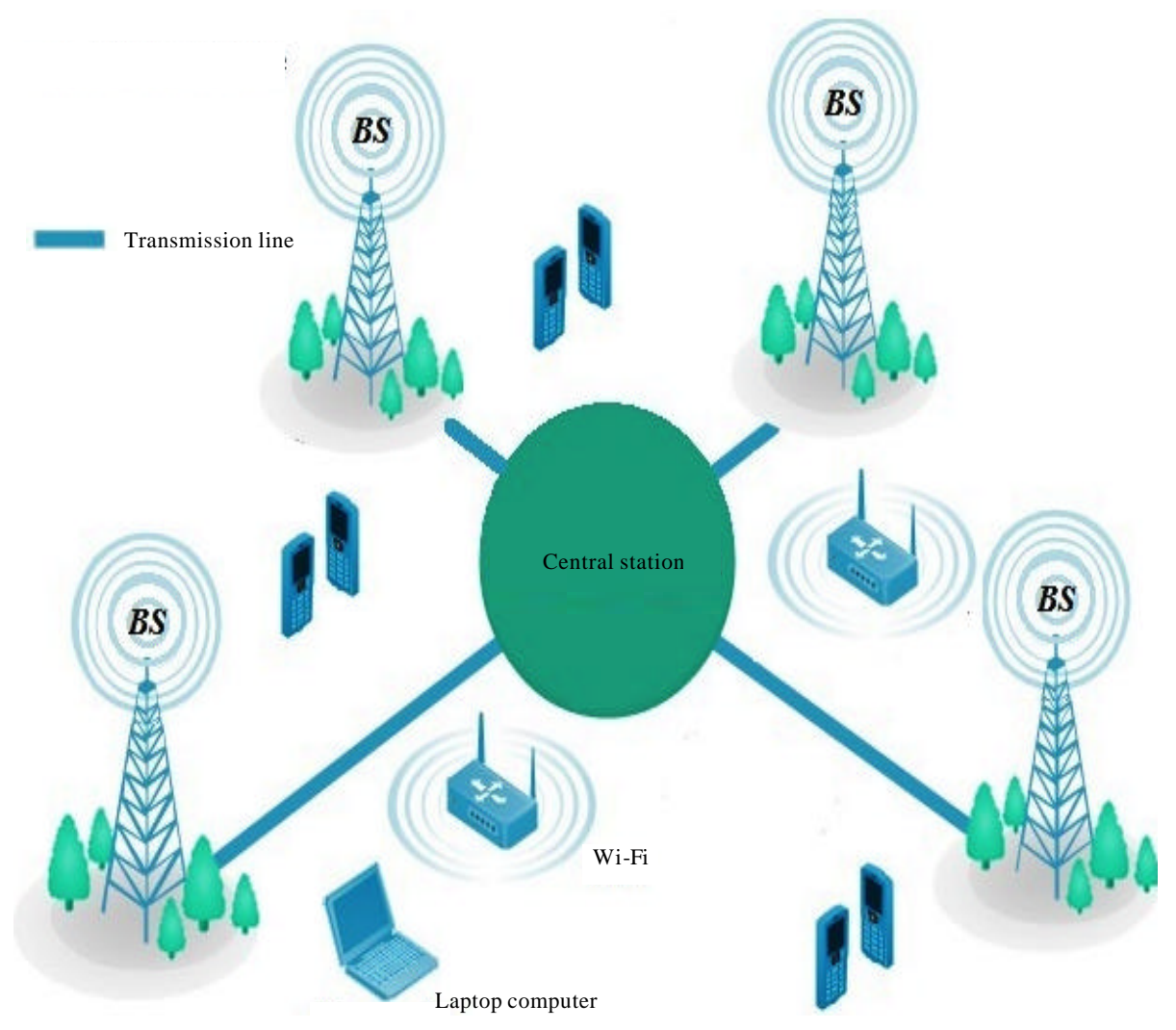

Fig. 1: Electrical transportation system for mm-W signals

high propagation loss of such waveguides or coaxial cables. Thus, the central generation and transmission of $\mathrm{mm}-\mathrm{W}$ wireless signals become impractical and that the data signals should be transmitted at a low Intermediate Frequency (IF) or a Baseband (BB) to BSs. At each BS, the IF or BB signal should be upconverted to the desired $\mathrm{mm}-\mathrm{W}$ signal, amplified and radiated. This process leads to an increase in BS complexity.

Figure 1 shows the typical electrical transportation system for mm-W wireless signals. The system composed of a switching center, BSs and a number of repeaters over the transmission lines to overcome signal degradation caused by noise and signal attenuation. The main drawback of this system is that the covered area from a switching center is restricted because of the degradation in the signal to noise ratio. Based on above discussion, we can deduce that the electrical approaches for the generation and transportation of modulated $\mathrm{mm}-\mathrm{W}$ signals are roughly limited in both cost and performance.

\section{OFU techniques}

Frequency doubling technique: This technique optically generates an mm-W frequency double that of the input
RF. This can be done by generating an Optical Double Sideband (ODSB) signal, i.e., optical carrier and two 1st order optical sidebands, after that filtering the optical carrier.

Frequency doubling using a single MZM without optical filter: This frequency doubling technique was developed by O'Reilly et al. (1992). An frequency-doubling was achieved by biasing a Dual Drive-MZM (DD-MZM) at Minimum Transmission Bias Point (MITBP). The component at $\omega_{\circ}$ (the optical carrier) will suppressed as well as the entire components at $\omega_{0} \pm 2 n \omega_{\mathrm{RF}}$. Thus, two strong 1 st order optical sidebands separated by $2 \mathrm{w}_{\mathrm{RF}}$ are obtained. This optical mm-W generation technique is immune to fiber CD because the generated $\mathrm{mm}-\mathrm{W}$ signal originates from the beat of the two strong 1 st order optical sidebands.

Frequency doubling without using DC bias adjustment: Chien and Hsueh proposed a technique to generate a frequency doubling optical mm-W signal by using an optical PM and a BGF (Chien et al., 2010). Phasemodulated carrier is generated by modulating the phase of a $\mathrm{CW}$ generated from a DFB-LD with a sinusoidal RF 
Table 1: Comparison of OFU techniques for optical generation and transmission of mm-W signals

\begin{tabular}{|c|c|c|}
\hline Technique & Advantages & Disadvantages \\
\hline Frequency doubling & $\begin{array}{l}\text { Simple configuration } \\
\text { Suitable for long distance transimission of the optical fiber }\end{array}$ & $\begin{array}{l}\text { Not adequate to minimize requirements on the electrical components } \\
\text { Low efficiency frequency upconversion }\end{array}$ \\
\hline Frequency quadrupling & $\begin{array}{l}\text { Cost effective } \\
\text { Low RF popwer is needed to achieve high OHDSR }\end{array}$ & $\begin{array}{l}\text { Not adequate to minimize requirements on the electrical components } \\
\text { for application at frequencies above } 100 \mathrm{GHz}\end{array}$ \\
\hline Frequency sixtupling & Driving $\mathrm{RF}$ at one sixth the required $\mathrm{mm}-\mathrm{W}$ frequency & $\begin{array}{l}\text { High modulation index requirement } \\
\text { Bias drifting problem } \\
\text { mm-W signal suffers from power fading caused by fiber CD }\end{array}$ \\
\hline Frequency octupling & $\begin{array}{l}\text { Driving } \mathrm{RF} \text { at one eight the required } \mathrm{mm}-\mathrm{W} \text { frequency } \\
\text { Adequate ti minimize requirements on the electrical requirements }\end{array}$ & $\begin{array}{l}\text { High modulation index requirement } \\
\text { Complicated } \\
\text { The mm-W signal is more susceptible to bit walk-Off eccect }\end{array}$ \\
\hline
\end{tabular}

signal by using PM. After filtering high-order optical sidebands by using a BGF with a bandwidth $\left(2-4 \omega_{m}\right)$, the phase modulated signal composed of the optical carrier, 1 st and 2nd order optical sidebands. The key benefit of using this technique is that the frequency doubling can be accomplished without particular DC bias adjustment thus eliminating the bias drifting difficulty (Qi et al., 2005). However, to remove the undesired optical sidebands, a BGF should be used. The BGF is sensitive to temperature. Moreover, most optical power will be lost because of BGF reflection (Yu et al., 2006) (Table 1).

\section{Frequency quadrupling technique \\ Frequency doubling using a single MZM without optical} filter: Qi et al. (2005) demonstrate a technique that can achieve quadruple-frequency optical $\mathrm{mm}-\mathrm{W}$ signal generation utilizing single MZM. The MZM is biased at the Maximum Bias Point (MATBP). By multiplying the 2nd order optical sidebands at a high-speed PIN-PD a quadruple-frequency $\mathrm{mm}-\mathrm{W}$ signal is generated. Nevertheless, the main problem in this technique is: the purity of the optical mm-W signal is low. Moreover, an ultra-narrowband optical filter is needed to vanish the optical-carrier, thus, resulting in a system that has poor stability and high cost.

Frequency quadrupling using two cascaded MZMs: By Mohamed et al. (2012) present a technique to generate quadruple frequency optical $\mathrm{mm}-\mathrm{W}$ signal: two cascaded DD-MZMs biased at the QBP with a phase shift $180^{\circ}$ between the two DD-MZMs. When the light is injected into the first DD-MZM, the optical carrier and upper 1st order optical sideband are generated while the lower 1st order sideband is suppressed. Then, the upper 1 st order optical sideband is removed after the second DD-MZM. Thus, only the optical carrier and the upper and lower 2nd order optical sidebands are generated after the second DD-MZM. However, the mm-W generation is caused by the multiplying of optical sidebands at $\pm 2 \omega_{\mathrm{RF}}$ and the multiplying of the optical carrier optical with sidebands at $\pm 2 \omega_{\mathrm{RF}}$, thus, producing destructive and constructive interactions to occur and causing fiber dispersion induced power fading on the desired mm-W (Al-Shareefi et al., 2013a, b).

Frequency quadrupling using two parallel MZMs: A frequency-quadrupling technique that can produce a high-purity $160 \mathrm{GHz}$ Optical Carrier Suppression (OCS) mm-W signal using two commercially available DDMZMs is developed by Al-Shareefi et al. (2013a, b). Two parallel DD-MZMs biased at the MITBP with a $90^{\circ}$ phase among the RF drive signals applied to every DD-MZM electrodes and a $180^{\circ}$ phase among the $\mathrm{RF}$ drive signals applied to the DD-MZMs are used. By using this technique an Optical Harmonic Distortion Suppression Ratio (OHDSR) more than $42 \mathrm{~dB}$ can be achieved. Therefore, high upconversion efficiency can be attained with less non-linear distortion effect. A typical modulation index $\mathrm{m}=2.4048$ is required to drive the MZMs that is cost-effective.

\section{Frequency sixtupling technique}

Frequency sixtupling using two cascaded DD-MZMs: By Qin and Sun (2012) present an approach to generate a sixfold frequency optical mm-W signal. This frequency sixtupling technique uses two DD-MZMs (MZM1 and MZM2) and a Gaussian Optical Band Pass Filter (GOBPF). MZM1 is biased at the MITBP whereas MZM2 is biased at MATBP and is used for modulating the data signal. Utilizing this technique, the required 3rd order optical sidebands are maximized while the unwanted optical sidebands are significantly removed. Hence, high efficiency frequency upconversion is achieved with minimum nonlinear distortion impact. The major limitation of this technique is the bias drift of DD-MZMs which makes the optical mm-W generation scheme a sophisticated control. Moreover, GOBF is required to achieve $\mathrm{mm}-\mathrm{W}$ signal generation, thus, resulting in a system that has poor stability (Zhang, 2011).

Frequency sixtupling using an integrated MZM: By Shi et al. (2011) present a new frequency sixtupling technique for the $\mathrm{mm}-\mathrm{W}$ signal generation. The frequency sixtupling technique uses an integrated MZM that is 
composed of three MZMs. The sub-MZMs are biased at MITBP. The phase difference among the two RF drive signals of the two sub-MZMs is $72^{\circ}$. Utilizing this technique an OHDSR higher than $29 \mathrm{~dB}$ can be obtained, i.e., high-purity optical mm-W signal generation. However, to obtain a high OHDSR, a modulation index $\mathrm{m}=3.8317$ is required to drive the IMZM, thus, suggesting that a high $\mathrm{RF}$ power is needed that is not cost-efficient.

\section{Frequency octupling technique}

Frequency octupling utilizing four nested MZMs: By Shang et al. (2012) proposes a filterless optical mm-W signal generation technique with frequency octupling utilizing four nested MZMs. The frequency octupling mmW generation system consists of two parallel frequency quadrupling systems. Each frequency quadrupling system is consist of two cascaded MZMs with a $90^{\circ}$ phase delay between the two RF signals. The proposed technique offers a high--quality optical mm-W generation, i.e., $\mathrm{OHDSR}=40 \mathrm{~dB}$ at $80 \mathrm{GHz}$. In addition, the system is unaffected by MZM bias drift which indicates a higher stability. However, complexity and cost are the major limitations of this technique.

Frequency octupling cutilizing two cascaded MZMs: By Chen et al. (2010) used two cascaded MZMs to produce optical mm-W signals with 8-fold input RF signal. The $\mathrm{mm}-\mathrm{W}$ generation technique is composed of two cascaded MZMs biased at the MATBP and an RF drive signal that is applied to the cascaded MZMs with a $-90^{\circ}$ phase shift. The technique minimizes the frequency demand of the oscillator and the modulator. However, the modulation efficiency of the technique is low. The modulation efficiency is the produced optical power measured after the optical modulator versus the input RF power (Lin el al., 2009a, b). Moreover, modulators have large insertion losses especially when cascaded (Alavi et al., 2016). Table 1 summarizes the drawbacks and benefits of the OFU techniques in this study.

\section{MATERIALS AND METHODS}

The flow chart that describes the research methodology is illustrated in Fig. 2. The main objective of the chart is to visualize input and output from the stages of the research for a better understanding of the evaluation of the OFU techniques.

Figure 2 provides an assessment of $O F U$ techniques based on the MZM for optical generation and the transmission of wireless signals mm-W. As depicated in Fig. 2, the flow chart consists of three parts:

Review of the OFU techniques: we start by reviewing specific OFU techniques that enable to double, quadruple, sixtuple or octuple an $R F$, gives a brief description of these techniques and highlighting obstacles and benefits.

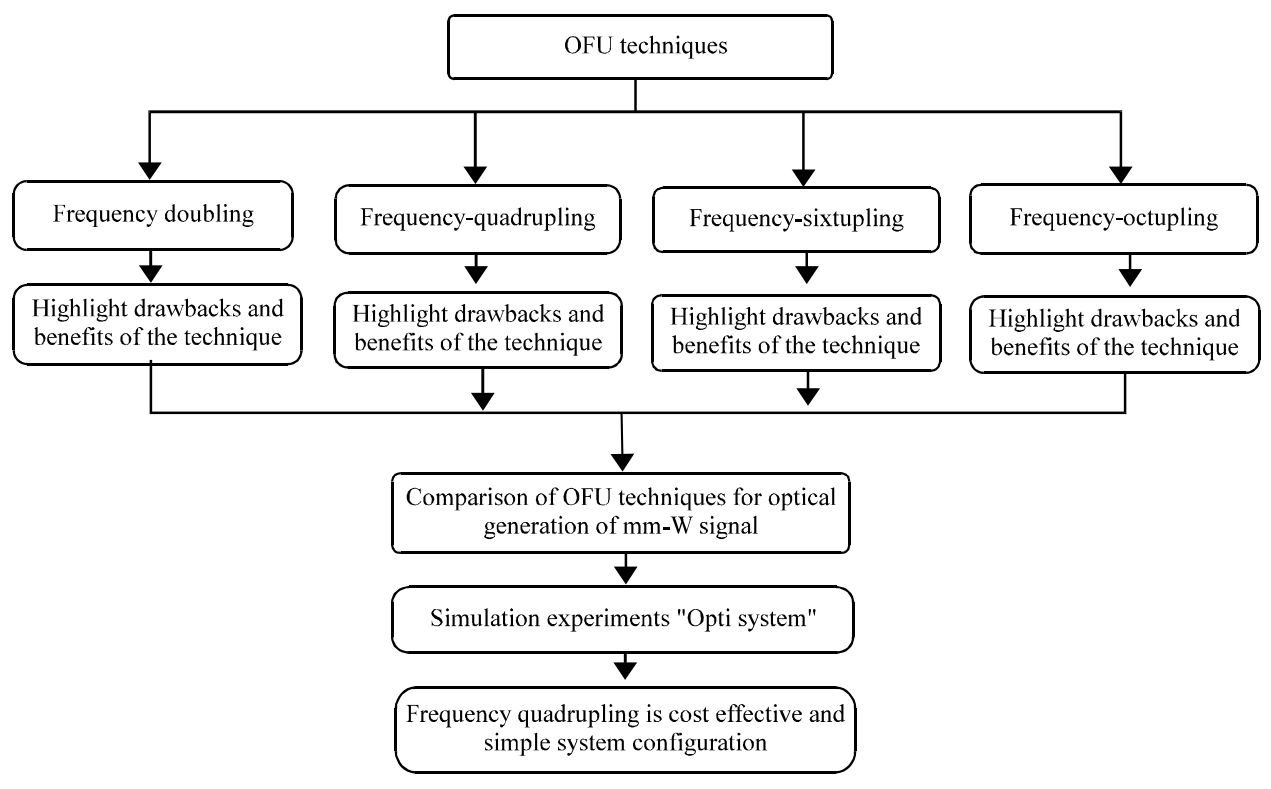

Fig. 2: Research methodology 
Nine OFU techniques were reviewed: two for frequency-doubling, frequency-sixtupling, frequencyoctupling and three for frequency-quadrupling.

Comparison of OFU techniques: Four types of OFU techniques is compared, namely, frequency-doubling, frequency-quadrupling, frequency-sixtupling and frequency-octupling. Comparison is made in terms of several parameters such as: modulation index, OHDSR, ESSR, bias drifting and modulation efficiency to show the trade-off between the complexity of OFU techniques and their costs.

Report the results: Computer simulation with "OptiSimTM 9.0" package have been carried out for the selected OFU techniques to assess the purity of the optical and RF mm-W signals. OHDSR and ESSR for the selected OFU techniques for diffent modulation index values have been compared. Additionally, the impact of phase shift, MZM extincation ratio and the frequency of the RF driving signal was also investegated. Simulation results showed that frequency-quadrupling was a more powerful and desirable option for optical generation and the transmission of mm-W wireless signals.

\section{RESULTS AND DISCUSSION}

Computer simulation with "OptiSim TM 9.0" package have been performed for the selected OFU techniques to assess the purity of the optical and RF mm-W signals when MZM ER is $100 \mathrm{~dB}$. The ER of $100 \mathrm{~dB}$ can be considered to be infinite.

Figure 3 shows the output optical and RF spectra for technique (O'Reilly et al., 1992), i.e., frequency-doubling.

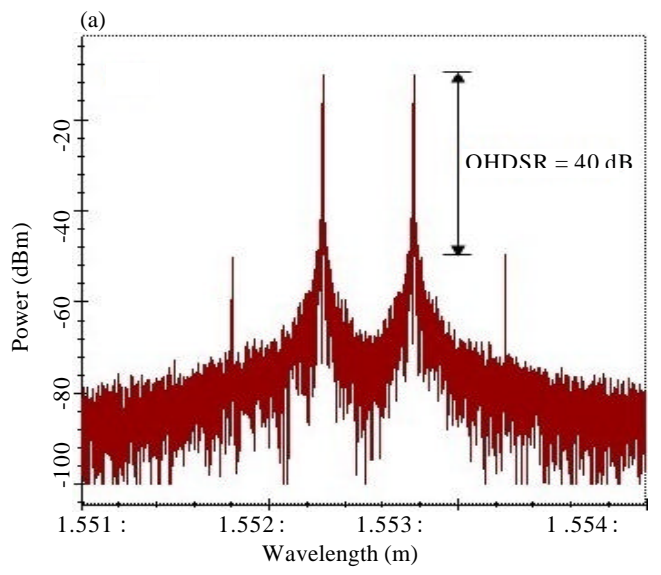

The power of the 1 st order sideband is $-10 \mathrm{dBm}$ and its OHDSR is $40 \mathrm{~dB}$ as depicted in Fig. 3a. The desired 60$\mathrm{GHz}$ and with $150 \mathrm{GHz}$ harrmonic mm-W signals are generated with an electrical spurious suppression ratio (ESSR) of $30 \mathrm{~dB}$ as depicted in Fig. 3b. The modulation index $\mathrm{m}=2.4048$. Figure 4 shows the output optical and RF spectra for technique (Al-Shareefi et al., 2013a, b), i.e., frequency-quadrupling. The power of the 2 nd order sideband is $-15 \mathrm{dBm}$ and its OHDSR is $40 \mathrm{~dB}$ as depicted in Fig. 4a. The required frequency quadrupling $60 \mathrm{GHz}$ and $120 \mathrm{GHz}$ harmonic mm-W signals are generated with an ESS of $35 \mathrm{~dB}$ as depicted in Fig. 4b. The modulation index $\mathrm{m}=2.4048$.

Figure 5 shows the output optical and RF spectra for technique (Shi et al., 2011) i.e., frequency-sixtupling. The power of the $3 \mathrm{rd}$ order sideband is $-20 \mathrm{dBm}$ and its OHDSR is $30.1 \mathrm{~dB}$ as shown in Fig. 5a. The $60 \mathrm{GHz}$ and specious $40 \mathrm{GHz}$ mm-W signals are generated with an ESSR of $25 \mathrm{~dB}$ as depicted in Fig. 5b. The modulation index $\mathrm{m}=3.831$.

Figure 6 shows the output optical and RF spectra spectra for technique (Shang et al., 2012) i.e., frequency-octupling. The power of the 4 th order sideband is $-20 \mathrm{dBm}$ and its OHDSR is $42 \mathrm{~dB}$ as depicted in Fig. $6 \mathrm{a}$. The RF spectrum comprises of a dc component and the 80 $\mathrm{GHz}$ and its ESSR is $44 \mathrm{~dB}$ as depicted in Fig. 6b. The modulation index $\mathrm{m}=3.831$.

Figures 3-6 reveal that the high purity optical mm-W signal can be produced with different modulation indexes. However, technique (Shi et al., 2011) and technique (Shang et al., 2012) require a high modulation index, i.e., require a high input $\mathrm{RF}$ power, i.e., not cost effective techniques.

In previous simulation analysis, a fixed ER is utilized. The OHDSR is affected by non-ideal MZM ER. To assess the impact of the alteration of MZM ER on OHDSR

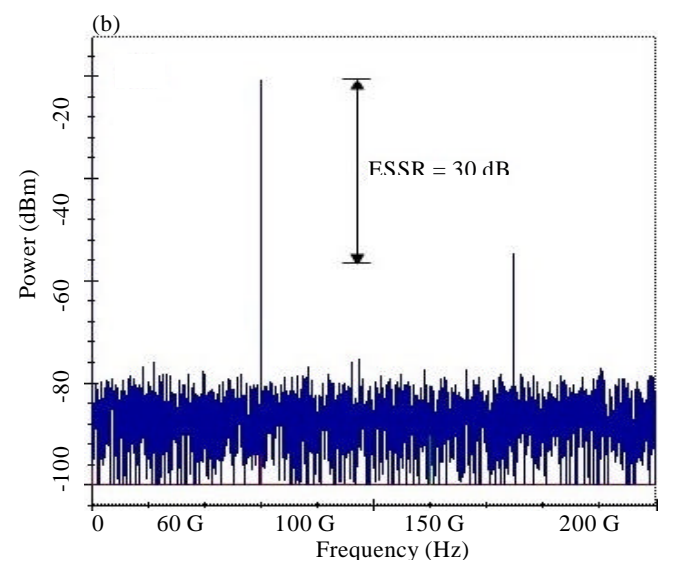

Fig. 3: a) Optical spectrum and b) RF spectrum. B-T-B case for Technique (O'Reilly et al., 1992). OHDSR = 40 $\mathrm{dB}, \mathrm{ESSR}=30 \mathrm{~dB}, \mathrm{~m}=2.4048, \mathrm{RF}$ oscillator $=30-\mathrm{GHz}$ 

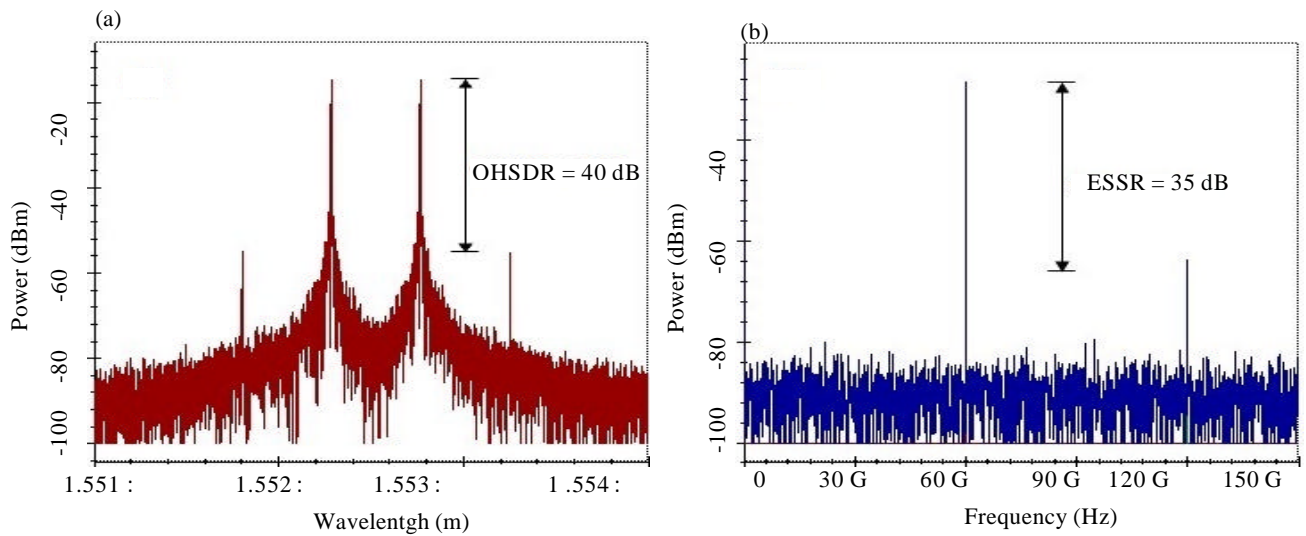

Fig. 4: a) Optical spectra and b) RF spectrum. B-T-B case for technique (Al-Shareefi et al., 2013a, b). OHDSR= $40 \mathrm{~dB}$, $\mathrm{ESSR}=35 \mathrm{~dB}, \mathrm{~m}=2.4048, \mathrm{RF}$ oscillator $=15-\mathrm{GHz}$
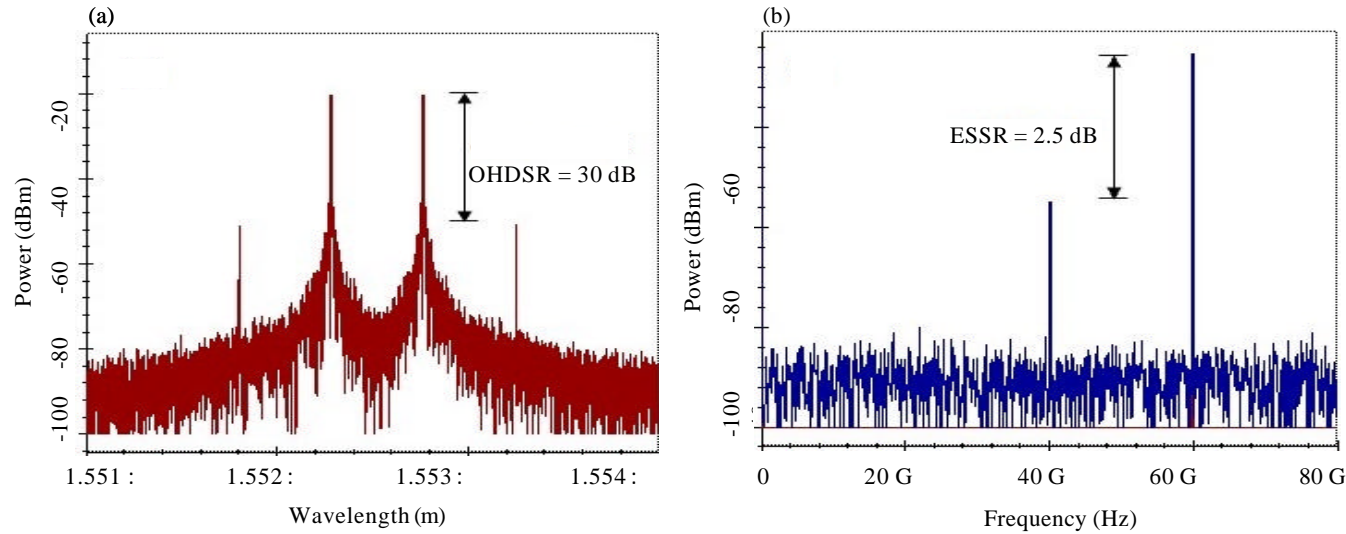

Fig. 5: a) Optical spectra and b) RF spectrum B-T-B case for technique (Shi et al., 2011). OHDSR $=30 \mathrm{~dB}, \mathrm{ESSR}=25 \mathrm{~dB}$, $\mathrm{m}=3.831, \mathrm{RF}$ oscillator $=10 \mathrm{GHz}$
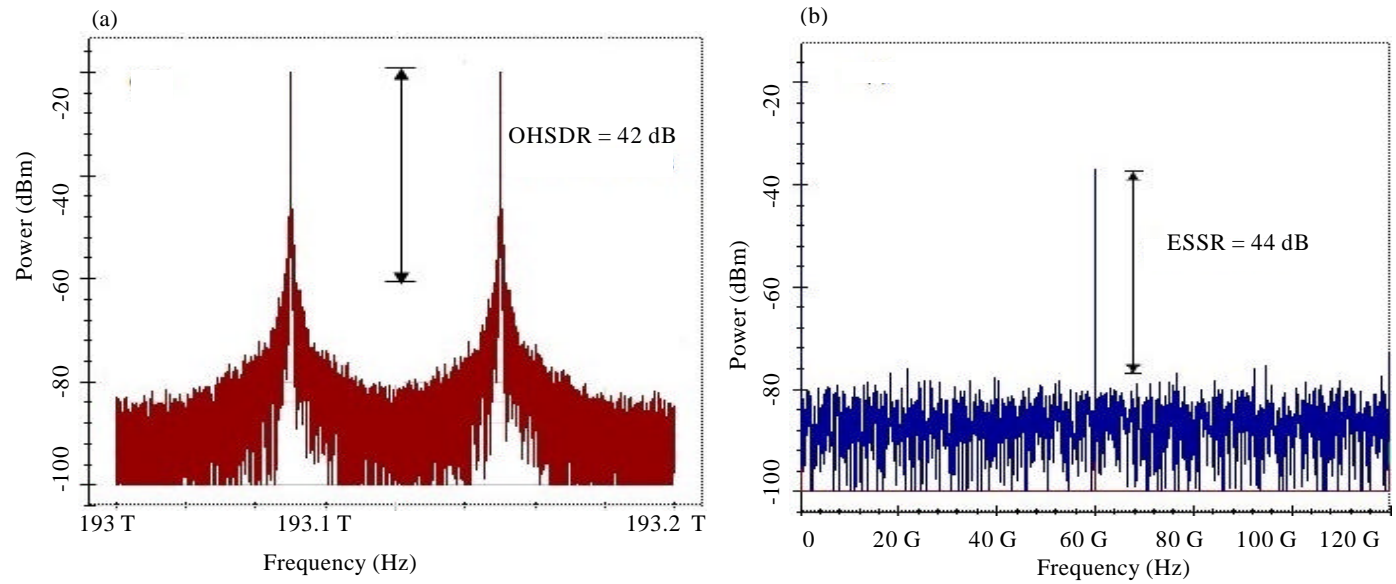

Fig. 6: a) Optical spectra and b) RF spectrum. B-T-B case for technique (Shang et al., 2012). $\mathrm{OHDSR}=40 \mathrm{~dB}, \mathrm{ESSR}=40$ $\mathrm{dB}, \mathrm{m}=3.831, \mathrm{RF}$ oscillator $=7.5-\mathrm{GHz}$

for the OFU techniques, the MZM ER is increased from $20-70 \mathrm{~dB}$ and its impact on OHDSR is depicted in Fig. 7.
Figure 7 shows that the value OHDSR for technique (Al-Shareefi et a l., 2013a, b) increases from $35 \mathrm{~dB}$ at ER of $20 \mathrm{~dB}$ and reaches its greatest value of $42 \mathrm{~dB}$ when $\mathrm{ER}$ 


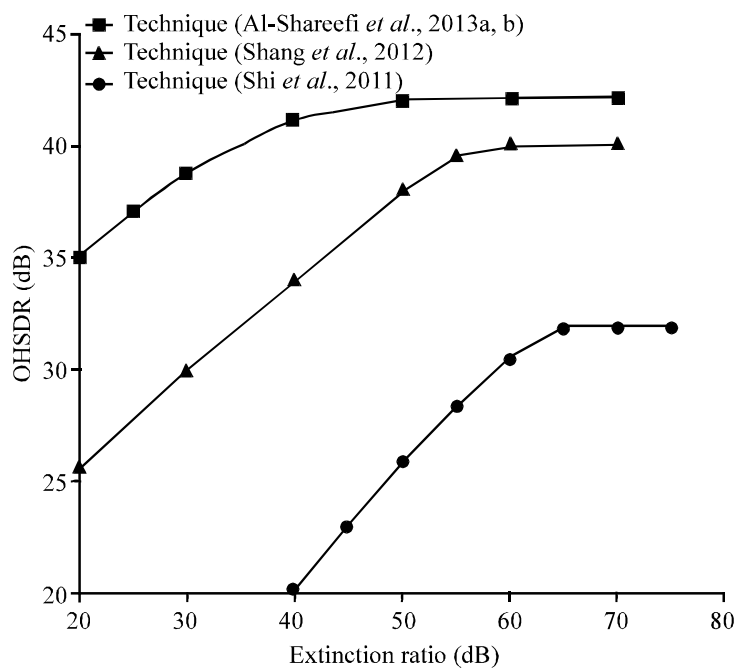

Fig. 7: Effect of non-ideal MZM ER on OHDSR

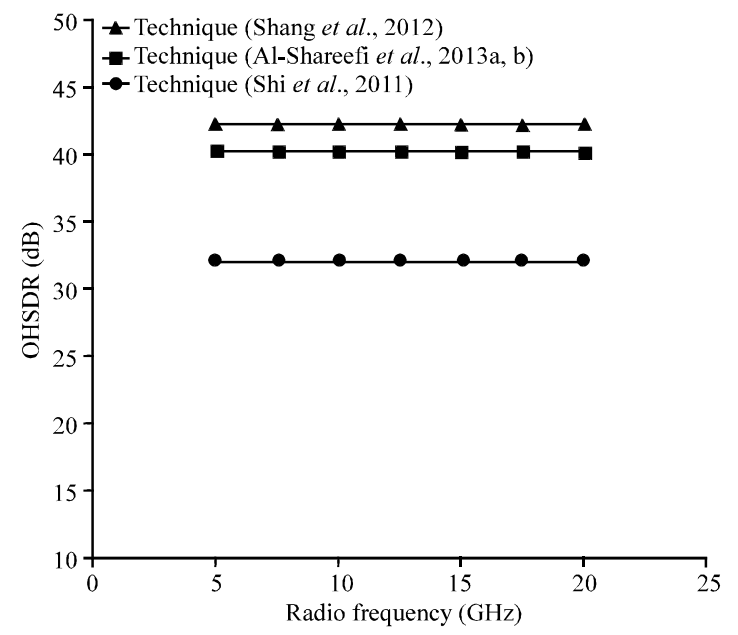

Fig. 8: Effect of RF oscillator on OHDSR

is $50 \mathrm{~dB}$, after which it will remain fixed while OHDSR for technique (Shi et al., 2011) increases from $20 \mathrm{~dB}$ at ER of $40 \mathrm{~dB}$ and it reaches greatest value of $32 \mathrm{~dB}$ when the $\mathrm{ER}$ is $65 \mathrm{~dB}$ then it stays fixed. OHDSR for technique (Shang et al., 2012) increases from $25 \mathrm{~dB}$ at $20 \mathrm{~dB}$ ER and reaches its greatest value of $41 \mathrm{~dB}$ when $\mathrm{ER}$ is $60 \mathrm{~dB}$ and then remains fixed.

The increase of the ER value will increase the power of the desired 2nd-4th order optical sidebands, i.e., increasing OHDSR and thereby ESSR. Note that technique (Al-Shareefi et al., 2013a, b) is nearly unchanged by the non-ideal MZM ER.

To access the impact of the alteration of the frequency of the RF driving signal on OHDSR for the OFU techniques, the frequency is changed from $7.5-22.5 \mathrm{GHz}$ and its impact on OHDSR is depcited in Fig. 8.

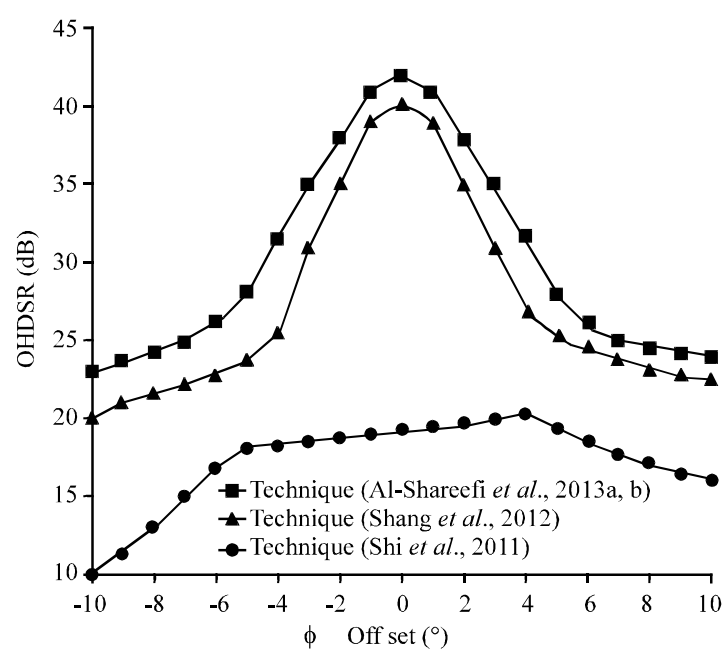

Fig. 9: Effect of phase shifting on OHDSR

For technique Al-Shareefi et al. (2013a, b) the generated wireless $\mathrm{mm}-\mathrm{W}$ signal alters from $30-90 \mathrm{GHz}$. For technique Shi et al. (2012) the generated wireless mm-W signal alters from 45-135 Ghz. For technique Shang et al. (2012) the generated wireless mm-W signal alters s from $60-180 \mathrm{GHz}$. It is clear from Fig. 8 that the OHDSR remain fixed at 40,32 and $42 \mathrm{~dB}$ for the techniques with the increase of frequency of the $\mathrm{RF}$ driving signal. Hence, the OFU techniques are independent of the alteration of the oscillator frequency.

To evaluate the impact of the alteration of phase shifting on OHDSR for the techniques, the phase shift value is changed from $-10-10^{\circ}$ and its impact on OHDSR is shown Fig. 9. For technique Al-Shareefi et al. (2013a, b). The highest OHDSR can be achieved for a phase shift near the $0^{\circ}$. The value then decreases slowly with the increment of the phase shift deviation value, i.e., $0^{\circ}$. An OHDSR greater than $24 \mathrm{~dB}$ can be achieved if the deviation is within $9^{\circ}$. The ER is equal to $25 \mathrm{~dB}$. For technique Shi et al. (2011) and technique Shang et al. (2012) an ER of 40 and $30 \mathrm{~dB}$ are utilized and OHDSR is susceptible to nonideal phase difference deviation.

\section{CONCLUSION}

This study has provided an evaluation of of the OFU techniques for generation and transportation of $\mathrm{mm}-\mathrm{W}$ wireless signals; highlighting the drawbacks and benefits of these techniques. The frequency-doubling technique is simply achieved with a single MZM. However, the frequency doubling technique is not adequate to minimize requirements on the electrical and optical components. Frequency-sixtupling and octupling techniques can 
minimize the demands significantly. However, strict demand on high modulation index is desired, i.e., not cost effective. Moreover, in the frequency sixtupling and octupling the conversion efficiency is small and the $\mathrm{mm}-\mathrm{W}$ signal suffers from os cillation in RF power and bit walk-off effect caused by the fiber dispersion. The frequency-quadrupling technique can be simply achieved by using a single MZM. But the frequency quadrupling technique is not adequate to minimize requirements on the electrical components for wireless applications at frequencies above $100 \mathrm{GHz}$. Therefore, frequency quadrupling technique is a more desirable choice to generate the $\mathrm{mm}-\mathrm{W}$ signal.

\section{REFERENCES}

Al-Shareefi, N.A., S.I.S. Hassan, M.F.B.A. Malek, R. Ngah and S.A. Aljunid et al., 2013a. Development of a new approach for high-quality quadrupling frequency optical millimeter-wave signal generation without optical filter. Prog. Electromagnet. Res., 134: 189-208.

Al-Shareefi, N.A., S.S. Hassan, F. Malek, R. Ngah and S.A. Aljunid et al., 2013b. A study in OCS millimeter-wave generation using two parallel DD-MZMs. Proceedings of the 2013 IEEE 11th Malaysia International Conference on Communications (MICC), November 26-28, 2013, IEEE, Kuala Lumpur, Malaysia, pp: 418-421.

Alavi, S.E., M.R.K. Soltanian, I.S. Amiri, M. Khalily, A. S.M. Supa'At and H. Ahmad, 2016. Towards 5G: A photonic based millimeter wave signal generation for applying in 5G access fronthaul. Sci. Rep., 6: 1-11.

Chen, Y., A. Wen and L. Shang, 2010. Analysis of an optical mm-wave generation scheme with frequency octupling using two cascaded mach-zehnder modulators. Opt. Commun., 283: 4933-4941.

Chien, H.C., Y.T. Hsueh, A. Chowdhury, J. Yu and G.K. Chang, 2010. On frequency-doubled optical millimeter-wave generation technique without carrier suppression for in-building wireless over fiber applications. IEEE. Photonics Technol. Lett., 22: 182-184.

Chuyanov, V., V. Kupershmidt, F. Kusnadi, H. Plaessmann and M. Ozcan, 2005. Packaged heterodyne source modules based on external cavity lasers for microwave applications. Terahertz Gigahertz Electron. Photonics IV, 5727: 82-88.

Gubbia, J., R. Buyya, S. Marusic and M. Palaniswami, 2013. Internet of Things (IoT): A vision, architectural elements and future directions. Future Generat. Comput. Syst., 29: 1645-1660.
Hyodo, M., S. Saito and Y. Kasai, 2009. Optical phase-locked loop with fibre lasers for low phase noise millimetre-wave signal generation. Electron. Lett., 45: 878-880.

Khawaja, B.A. and M.J. Cryan, 2008. Characterization of multimode fibers for use in millimeter wave radio-over-fiber systems. Microwave Opt. Technol. Lett., 50: 2005-2007.

Kitayama, K.I., T. Kuri, H. Yokoyama and M. Okuno, 1996. $60 \mathrm{GHz}$ millimeter-wave generation and transport using stabilized mode-locked laser diode with optical frequency DEMUX switch. Proceedings of the GLOBECOM'961996 International Conference on Global Telecommunications, Communications: The Key to Global Prosperity Vol. 3, November 18-28, 1996, IEEE, London, UK, pp: 2162-2169.

Kuri, T., K.I. Kitayama and Y. Takahashi, 2003. A single light-source configuration for full-duplex 60-GHz-band radio-on-fiber system. IEEE. Trans. Microwave Theor. Tech., 51: 431-439.

Li, X., J. Xiao and J. Yu, 2016. Long-distance wireless mm-wave signal delivery at W-band. J. Lightwave Technol., 34: 661-668.

Lin, C.T., P.T. Shih, J. Chen, W.J. Jiang and S.P. Dai et al., 2009a. Optical millimeter-wave up-conversion employing frequency quadrupling without optical filtering. IEEE. Trans. Microwave Theor. Tech., 57: 2084-2092.

Lin, C.T., P.T. Shih, W.J. Jiang, J.J. Chen, P.C. Peng and S. Chi, 2009b. A continuously tunable and filterless optical millimeter-wave generation via. frequency octupling. Opt. Express, 17: 19749-19756. Mohamed, M., X. Zhang, B. Hraimel and K. Wu, 2007. Efficient photonic generation of millimeter-waves using optical frequency multiplication in radio-over-fiber systems. Proceedings of the IEEE International Topical Meeting on Microwave Photonics, October 3-5, 2007, IEEE, Victoria, Canada, ISBN:978-1-4244-1167-2, pp: 179-182.

Mohamed, M., X. Zhang, B. Hraimel and K. Wu, 2012. Impact of modulation index on transmission performance of millimeter wave multiband OFDM ultra-wide-band wireless signal over fiber system. Photonics North Intl. Soc. Opt. Photonics, 8412: 84120S-84120S.

O'Reilly, J.J., P.M. Lane, R. Heidemann and R. Hofstetter, 1992. Optical generation of very narrow linewidth millimetre wave signals. Electron. Lett., 28: 2309-2311. 
Qi, G., J. Yao, J. Seregelyi, S. Paquet and C. Belisle, 2005. Generation and distribution of a wide-band continuously tunable millimeter-wave signal with an optical external modulation technique. IEEE. Trans. Microw. Theory. Tech., 53: 3090-3097.

Qin, Y. and J. Sun, 2012. Frequency sextupling technique using two cascaded dual-electrode Mach-Zehnder modulators interleaved with Gaussian optical band-pass filter. Opt. Commun., 285: 2911-2916.

Rappaport, T.S., Y. Xing, G.R. MacCartney Jr, A.F. Molisch, E. Mellios and J. Zhang, 2017. Overview of millimeter wave communications for 5 th-generation $(5 \mathrm{G})$ wireless networks-with a focus on propagation models. IEEE. Trans. Antennas Propag., 65: 6213-6230.
Shang, L., A. Wen, B. Li, T. Wang, Y. Chen and M.A. Li, 2012. A filterless optical millimeter-wave generation based on frequency octupling. Intl. J. Light Electron Opt., 123: 1183-1186.

Shi, P., S. Yu, Z. Li, S. Huang and J. Shen et al., 2011. A frequency sextupling scheme for high-quality optical millimeter-wave signal generation without optical filter. Opt. Fiber Technol., 17: 236-241.

Yu, J., Z. Jia, L. Yi, Y. Su, G.K. Chang and T. Wang, 2006. Optical millimeter-wave generation or up-conversion using external modulators. IEEE Photonics Technol. Lett., 18: 265-267.

Zhang, Y., 2011. Development of millimeter-wave radio-over-fiber technology. J. Electron. Sci. Technol., 9: 58-66. 